\title{
Nanoplasmonic laser fusion response to Földes and Pokol
}

L. P. Csernai ${ }^{1}$ (D), N. Kroó ${ }^{2,3}$, I. Papp ${ }^{2}$ and D. D. Strottman ${ }^{4}$

\section{Letter to the Editor}

Cite this article: Csernai LP, Kroó N, Papp I, Strottman DD (2020). Nanoplasmonic laser fusion response to Földes and Pokol. Laser and Particle Beams 38, 285-286. https:// doi.org/10.1017/S0263034620000348

Received: 24 August 2020

Revised: 28 August 2020

Accepted: 31 August 2020

First published online: 6 October 2020

\section{Key words:}

Inertial confinement fusion; nanoplasmonics; reaction rate; relativistic fluid dynamics

Author for correspondence: L. P. Csernai, Department of Physics and Technology, University of Bergen, Norway.

E-mail: laszlo.csernai@uib.no (c) The Author(s) 2020. Published by Cambridge University Press

\section{CAMBRIDGE UNIVERSITY PRESS}

${ }^{1}$ Department of Physics and Technology, University of Bergen, Bergen, Norway; ${ }^{2}$ Wigner Research Centre for Physics, Budapest, Hungary; ${ }^{3}$ Hungarian Academy of Sciences, Budapest, Hungary and ${ }^{4}$ Los Alamos National Laboratory, Los Alamos, NM, USA

\section{Abstract}

Földes and Pokol in their letter "Inertial fusion without compression does not work either with or without nanoplasmonics" criticized our works. Here, we refute their argumentation. Our proposed improvement is the combination of two basic research discoveries: (i) the possibility of detonations on space-time hypersurfaces with time-like normal (i.e., simultaneous detonation in a whole volume) and (ii) to increase the ignition volume to the whole target, by regulating the laser light absorption using nanoantennas. These principles can be realized in an in-line, one-dimensional configuration, in the simplest way with two opposing laser beams as in particle colliders.

First of all, the title of the letter of Földes and Pokol is misleading. Already in the abstract of Csernai et al. (2018), it is stated that we need to achieve limited compression to avoid the Rayleigh-Taylor instabilities. Furthermore, it is written that the aim is to achieve fusion "without too much pre-compression". It is false from Földes and Pokol to state in their conclusion that we "...misjudged the possibility of the application of the uncompressed inertial fusion scheme". We have never had such a statement.

In Csernai et al. (2018), the Lawrence Livermore National Laboratory (LLNL), National Ignition Facility (NIF) compression was discussed in some detail just as it is done by Földes and Pokol (2020). The difficulties of this NIF setup by an ablator are elaborated in both of these publications.

Földes and Pokol does not mention our work, where the improvements we propose are elaborated in greater detail (Csernai et al., 2020), and the experimental work showing that with the simplified linear irradiation from both sides can achieve the same compression as reached at LLNL NIF (Zhang et al., 2019). These works provide a theoretical and experimental response to the comments of Földes and Pokol.

In recent years, the laser wakefield acceleration (LWFA) became a well-known concept with useful applications. An intensive laser pulse impinging on a target creates a high-density plasma of $4 \times 10^{19} \mathrm{~cm}^{-3}$, and a wakefield wave follows the pulse. This nonlinear wave in dense plasma is formed of the EM-field, electrons, and ions. A typical laser pulse of $20 \mathrm{~mJ}$ intensity, $7 \mathrm{fs}$ length, and $\lambda$ wavelength can create a laser wakefield (LWF) dense plasma wave of about $10 \lambda$ wavelength. Electrons do not prevent the development of this LWF waves. In the configuration presented in Csernai et al. (2020) and Zhang et al. (2019), such LWF waves develop and move opposite to each other like in accelerators in collider configuration. This is suggested in Csernai et al. (2020) for laser-driven fusion. Here, two known effects were combined. First, the possibility of detonations on space-time hypersurfaces with time-like normal, so-called time-like detonations (Csernai, 1987; Csernai and Strottman, 2015), which were found theoretically and experimentally in high-energy heavy-ion collision in the couple of last decades. This simultaneous volume ignition eliminates the possibility of Rayleigh-Taylor instabilities, which is a serious obstacle in achieving laboratory scale nuclear fusion. The second effect we use is to achieve simultaneous detonation in the whole volume of the target, by regulating the laser light absorption with nanoshells or nanorods. This second effect is already experimentally proven in validation experiments, at Wigner RCP, Budapest, the Institute of Földes (Kroó and Rácz, 2016).

Földes and Pokol under points 1., 2., 3., describe the thermonuclear setup of ICF where a dense hot-spot is created in $\approx 10-20 \mathrm{~ns}$, in the middle of the target, and then, the flame front "slowly" propagates with $\alpha$-heating to the outside radius, $R$, of the target. As we emphasize in all previous publications, we do not follow this dynamics, instead we aim to reach the condition for ignition in $\approx 10-100 \mathrm{fs}$, in most of the target volume, that is, on a time-like hypersurface. Then, the flame front does not have to propagate anywhere, only the local burning rate matters! In the short ignition time, the ions cannot expand.

This was also emphasized (even in the title) in the above mentioned, published experiment using colliding laser beams (Zhang et al., 2019) achieved high target density for nuclear fusion. On the other hand, they did not utilize LWF waves and nanoplasmonics and did not attempt 
to have simultaneous transition in the target. Thus, our present proposal differs from this experiment in the two novel aspects.

In the publications commented on by Földes and Polkol, we focused on our two fundamental and novel ideas and did not discuss the aspects, which should not be modified. In laser wakefield collider (LWFC), the target has two sides, which are initially accelerated toward each other in a pre-acceleration and precompression process, on a nanosecond time scale, just like at LLNL NIF and in Zhang et al. (2019). The LWFA may reach the $\mathrm{GeV} /$ nucleon energy range.

In Zheng et al. (2011), it was shown that intense laser pulse irradiating a combination target can accelerate carbon ions to the $\mathrm{TeV}$ level by the laser plasma wakefield.

If we consider LWFC with a double layer pre-compressed to ion density, $n_{\mathrm{pc}}$, and pre-accelerated to several $\mathrm{GeV} /$ nucleon energy (i.e., to a velocity near to the speed of light, $v_{\mathrm{LWFC}} \approx c$ ), the two LWF waves can inter penetrate and lead to an ignition reaction rate of

$$
2 c \gamma^{2} n_{\mathrm{pc}}^{2} \sigma
$$

due to the Lorentz contraction of the two ion bunches to $\gamma n_{\mathrm{pc}}$, and where $\sigma$ is the ion-ion cross section. This may well exceed the thermal rate of $n\left\langle v_{\text {th }} \sigma\right\rangle$. If we accelerate the ions to $5 \mathrm{GeV} /$ nucleon, then $\gamma \approx 6$, and if we achieve a pre-compression of factor 8 (considerably less than at NIF, where the 3D compression reaches $\left.800 \mathrm{~g} / \mathrm{cm}^{3}\right)$, then our burning rate is $2 \cdot \gamma^{2} \frac{8^{2} n^{2}}{800 n}\left(c / v_{\text {th }}\right) \approx 270$ times bigger than at NIF. Here, we assumed that the average thermal collision speed is $v_{\text {th }} \approx c / 2$.

So, the non-thermal nonequilibrium LWFC mechanism may well exceed the thermal ignition rate by the adiabatic compression and heating at NIF. Especially, if this ignition at NIF takes place for a central hot-spot only and then the flame should propagate over the rest of the target. The extreme energy need mentioned at the end of point 3., arises from the assumption of the thermal, setup, that we do not follow.

According to our preliminary calculations (yet unpublished), we can see that at around 125-150 fs after the target and projectile touch, the two LWF waves constructively interact and the EM-field strength is maximal. This moment of time would be adequate for a short, intensive ignition pulse.

As we increase the target density the EM-field penetration into the target is reduced, as the energy and momentum are passed to the target and projectile ions. The longitudinal momentum is transported to the kinetic motion of the target particles, which then contribute to the pinch effect reducing the EM strength and target beam directed momentum.

Under points 4., and 5., the target opacity and absorptivity are discussed. Here, we want to emphasize that our nanoantennas are embedded within the target, so all energy reaching these remain in the target, so it is also absorbed. We have no extreme losses that occur due to evaporating the external hohlraum. The conversion of the absorbed energy to higher frequencies happens within the nanoantennas or can lead to direct nuclear burning.

Under point 6., Földes and Pokol assumes that the electrons will remain at the surface of the target. This fully contradicts with the structure of the LWF waves where the electrons are well separated from the ions. The electrons within the target are concentrated in the plasmonic waves on the surface of the nanoantennas. There is no significant electron reflection observed in LWFA, and even if, these electrons would just again remain in the target fuel in the LWFC configuration.

The conclusion of Földes and Pokol that "Therefore the nanostructures inside the target are of no use at all" is already proven to be false, as shown in the lower energy validation experiments at Wigner RCP, with laser beams of up to $25 \mathrm{~mJ}$ pulse energy, and polymer targets, which exhibit phase or structural transition at given threshold energy. This is also discussed already in Csernai et al. (2020) before the experiments were performed.

Summarizing the presented results both theoretically, and based on recent experiments (both in fusion related and highenergy heavy-ion fields), we can state that the conclusions of Földes and Pokol are unsubstantiated.

\section{References}

Csernai LP (1987) Detonation on timelike front for relativistic systems, School of Physics, University of Minnesota, Minneapolis, Minnesota, USA. Zhurnal Eksperimentalnoi I Teoreticheskoi Fiziki 92, 397-386; Soviet Physics - JETP 65, 219.

Csernai LP and Strottman DD (2015) Volume ignition via time-like detonation in pellet fusion. Laser and Particle Beams 33, 279-282.

Csernai LP, Kroó N and Papp I (2018) Radiation-dominated implosion with nano-plasmonics. Laser and Particle Beams 36, 171-178.

Csernai LP, Csete M, Mishustin IN, Motornenko A, Papp I, Satarov LM, Stöcker H and Kroó N (2020). Radiation-dominated implosion with flat target. Physics of Wave Phenomena 28, 187-199.

Földes IB and Pokol GI (2020) Inertial fusion without compression does not work either with or without nanoplasmonics. Laser and Particle Beams 39, 1-3.

Kroó N and Rácz P (2016) Plasmonics - The interaction of light with metal surface electrons. Laser Physics 26, 084011.

Zhang G, Huan M, Bonasera A, Ma YG, Shen BF, Wang HW, Xu JC, Fan GT, Fu HJ, Xue H, Zheng H, Liu LX, Zhang S, Li WJ, Cao XG, Deng XG, Li XY, Liu YC, Yu Y, Zhang Y, Fu CB and Zhang XP (2019) Nuclear probes of an out-of-equilibrium plasma at the highest compression. Physics Letters A 383, 2285-2289.

Zheng FL, Wu SZ, Zhou CT, Wang HY, Yan XQ and He XT (2011) An ultra-short and $\mathrm{TeV}$ quasi-monoenergetic ion beam generation by laser wakefield accelerator in the snowplow regime. Europhysics Letters (EPL) 95, 55005 . 